\title{
Estimating averted COVID-19 cases, hospitalisations, intensive care unit admissions and deaths by COVID-19 vaccination, Italy, January-September 2021
}

Chiara Sacco ${ }^{1}$, Alberto Mateo-Urdiales ${ }^{1}$, Daniele Petrone ${ }^{1}$, Matteo Spuri ${ }^{1}$, Massimo Fabiani ${ }^{1}$, Maria Fenicia Vescio ${ }^{1}$, Marco Bressi $^{1}$, Flavia Riccardo ${ }^{1}$, Martina Del Manso ${ }^{1}$, Antonino Bella ${ }^{1}$, Patrizio Pezzotti ${ }^{1}$, on behalf of the Italian Integrated Surveillance of COVID-19 study group ${ }^{2}$

1. Department of Infectious Diseases, Istituto Superiore di Sanità, Rome, Italy

2. The members of the Italian Integrated Surveillance of COVID-19 study group are acknowledged at the end of the article

Correspondence: Chiara Sacco (chiara.sacco@iss.it)

Sacco Chiara, Mateo-Urdiales Alberto, Petrone Daniele, Spuri Matteo, Fabiani Massimo, Vescio Maria Fenicia, Bressi Marco, Riccardo Flavia, Del Manso Martina, Bella Antonino, Pezzotti Patrizio, on behalf of the Italian Integrated Surveillance of COVID-19 study group. Estimating averted COVID-19 cases, hospitalisations, intensive care unit admissions and deaths by COVID-19 vaccination, Italy, January-September 2021. Euro Surveill. 2021;26(47):pii=2101001. https://doi. org/10.2807/1560-7917.ES.2021.26.47.2101001

We assessed the impact of COVID-19 vaccination in Italy, by estimating numbers of averted COVID-19 cases, hospitalisations, ICU admissions and deaths between January and September 2021, by age group and geographical macro areas. Timing and speed of vaccination programme implementation varied slightly between geographical areas, particularly for older adults. We estimated that 445,193 (17\% of expected; range: $331,059-616,054)$ cases, 79,152 (32\%; range: 53,209-148,756) hospitalisations, 9,839 ICU admissions (29\%; range: $6,434-16,276)$ and $22,067(38 \%$; range: $13,571-48,026)$ deaths were prevented by vaccination.

The roll-out of the vaccination against coronavirus disease (COVID-19) was launched in Italy on 27 December 2020 [1] and by the end of September 2021, 84\% of the eligible population aged 12 years and older, had received at least one dose of a vaccine against COVID19. National $[2,3]$ and international [4] researchers have found high levels of vaccine effectiveness (VE) against severe acute respiratory syndrome coronavirus (SARSCoV-2) infection and severe COVID-19.

We evaluated the direct impact of the Italian vaccination programme on the number of cases, on hospitalisations, on admissions to intensive care units (ICU) and on deaths, by estimating the numbers of these outcomes prevented (averted events) by COVID-19 vaccination between January (week 2/2021) and the end of September 2021 (week 38/2021) by age groups and geographical macro area.

\section{Vaccine deployment and uptake}

The target groups for COVID-19 vaccination followed the recommendations of the Ministry of Health [5], with healthcare workers, residents in long-term care facilities and persons aged over 80 years being the first to receive the vaccines. Successively, the vaccine rollout was extended to clinically extremely vulnerable groups and younger age groups in descending order, prioritising those with multiple comorbidities. The present vaccination programme in Italy targets the whole population aged 12 years and older with access to the national healthcare. About $80 \%$ of the vaccinated population has received the mRNA vaccines Cominarty (BNT162b2 mRNA, BioNTech-Pfizer, Mainz, Germany/ New York, United States (US)) or Spikevax (mRNA-1273, Moderna, Cambridge, US), whereas the rest of the population has received Vaxzevria (ChAdOx1 nCoV-19, Oxford-AstraZeneca, Cambridge, United Kingdom (UK) or COVID-19 Vaccine Janssen (Ad26.COV2-S, JanssenCilag International NV, Beerse, Belgium).

There was notable heterogeneity in the pace of vaccine uptake both across Italian regions and across Italian macro areas (North-West, North-East, Centre, and South-Islands, based on nomenclature of territorial units for statistics (NUTS1) areas for Italy [6]). While vaccine uptake was faster in the Centre of Italy, particularly in those aged 60-79 years, the South-Islands area has consistently reached lower levels of vaccine uptake in those aged 80 years and oldercompared the other macro areas (Figure 1). By the end of September (week 38), 65\% (ranging from 63\% in the North-East to $66 \%$ in the North-West and in the Centre) of those aged under 60 years, $84 \%$ (ranging from $82 \%$ in the South-Islands to $87 \%$ in the Centre) of those aged between 60 and 69 years, $89 \%$ (ranging from $87 \%$ in 


\section{FIGURE 1}

Cumulative monthly full vaccination coverage by age group and geographical macro area ${ }^{a}$, Italy, week 2/2021-week 38/2021

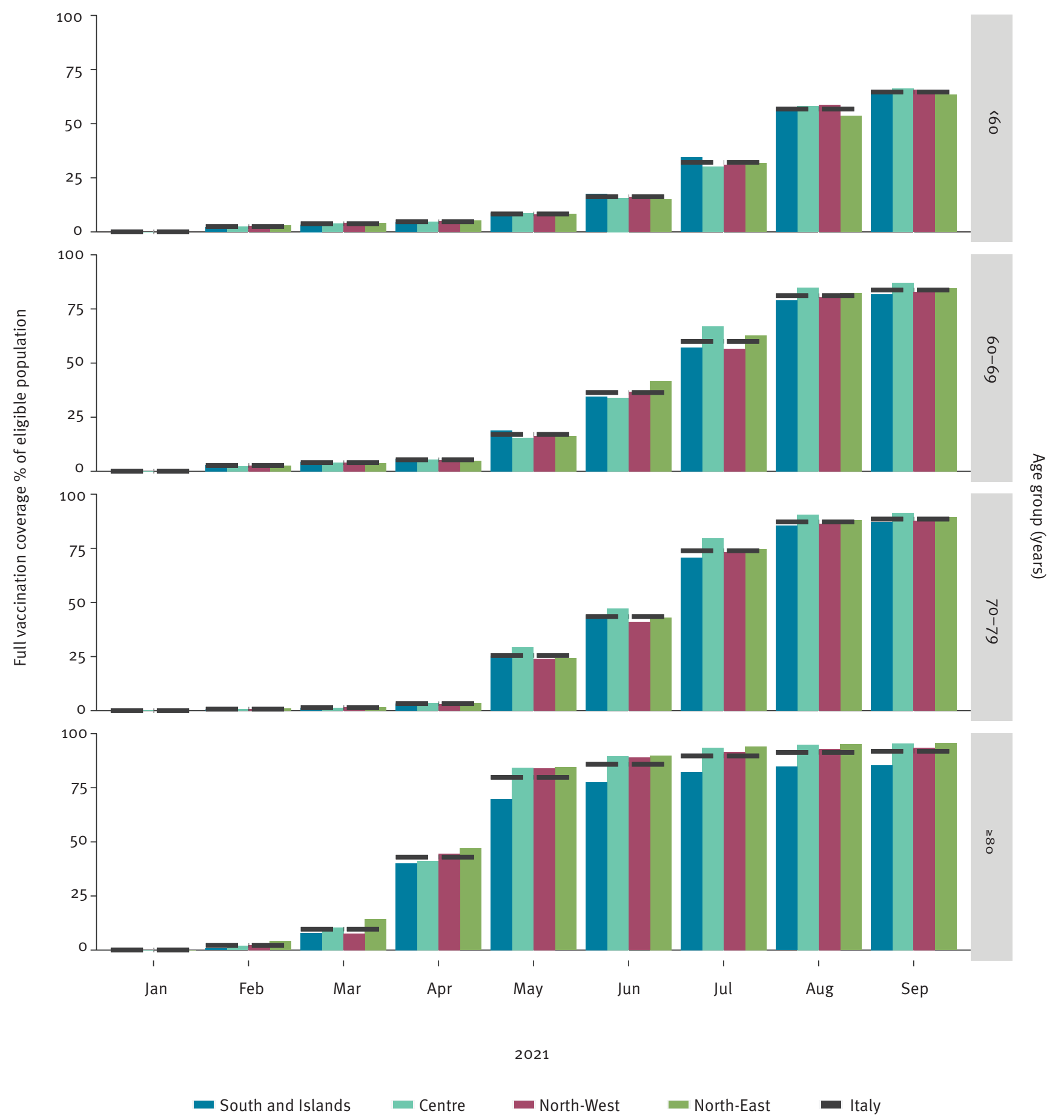

NUTS: nomenclature of territorial units for statistics.

a Based on NUTS1 areas for Italy [6]. 
the South-Islands to $91 \%$ in the Centre) of those aged between 70 and 79 years and 92\% (ranging from $85 \%$ in the South-Islands to and $96 \%$ in the North-East) of those aged 80 years and older had received the recommended number of doses of vaccine.

To account for the time-lag between vaccination and the development of immunity, we assumed a delay of 2 weeks for each of the vaccine doses [7,8]. Thus, we defined as partially vaccinated those in the period between 14 days post-first dose and 13 days postsecond dose; and as fully vaccinated those who had received the second dose or a single dose least 14 days earlier.

\section{Estimation of events averted by the vaccination programme}

To measure the events adverted, we obtained data on all notified COVID-19 cases exploiting the casebased national COVID-19 integrated surveillance system [9], and data from vaccine coverage through the national vaccination portal of the Ministry of Health [10], both updated on 11 November 2021. We focused on data covering the population aged 12 years and older, for the period between 11 January (week 2) and 30 September (week 38) 2021. The weekly number of COVID-19 cases, hospitalisations, ICU admissions and deaths averted by the vaccination campaign was estimated using a method widely used in the study of the impact of the vaccination during the influenza season $[11,12]$ and recently applied to calculate vaccine-prevented COVID-19 deaths [13]. Details can be found in the Supplementary Material 2.

The weekly number of observed COVID-19 cases, hospitalisations, ICU admissions and deaths were summarised by date of diagnosis or sampling since we were interested in measuring the number of cases hospitalised, admitted to ICU and died and not when these events took place. We included in our analysis only hospitalisations, ICU admissions and deaths that occurred within 30 days of the COVID-19 diagnosis, which account for ca $96 \%, 97 \%$, and $88 \%$ of the total numbers reported in the study period, respectively (Supplementary Figure S1). All analyses were stratified by age group ( 60 years, 60-69 years, 70-79 years, and 80 years and older), and geographical macro area. The results were further analysed by splitting the study period into three phases (January-March, April-June, July-September) characterised by different epidemiological situations and different levels of vaccination coverage (Table S1).

Details about VE estimation, methods and results used in this study can be found in the Supplementary Material 3. We also performed a sensitivity analysis varying the VE in an interval of $+/-10$ percentage points, considering as max upper limit $100 \%$. The results of the sensitivity analyses are presented as ranges of the estimated averted events to indicate uncertainties.
All the analysis were performed using R (version 4.1.1) [14]. The list of the $R$ packages used is available in the Supplementary Material.

\section{COVID-19 cases, hospitalisations, ICU admissions and deaths observed and averted}

A total of 445,193 (range: 331,059-616,054) cases, 79,152 (range: 53,209-148,756) hospitalisations, 9,839 (range: 6,434-16,276) ICU admissions and 22,067 (range: 13,571-48,026) deaths were estimated to have been averted by the vaccination campaign (Table), which account for $17 \%$ (range: $14 \%-23 \%$ ), $32 \%$ (range: $24 \%-47 \%$ ), $29 \%$ (range: $21 \%-41 \%$ ) and $38 \%$ (range: $28 \%-57 \%$ ) of the expected events (observed plus averted), respectively.

Age-stratified hospitalisations, ICU admissions and deaths

Without vaccination, the expected hospitalisation rate would have been $214,595,871,1,592$ per 100,000 respectively for those aged under 60, 60-69, 70-79 and 80 years and oldervs the observed rate of 163 , 421, 618, 886 per 100,000 (ranges see Table). In terms of admissions to ICU, we observed a differences of 5 (range: 4-6), 37 (range: 24-48), 50 (range: 31-80) and 50 (range: 30-128) events per 100,000 between the expected and the observed cumulative rate among those under 60, 60-69, 70-79 years old and those aged 80 years and older, respectively. We estimated that $71 \%$ (range: $69-79$ ) of the overall deaths were averted for those aged 80 years and older, and that $18 \%$ (range: $14-19$ ), $8 \%$ (range: $5-9$ ) and $2 \%$ (range: 1-3) were averted for those aged 70-79, 60-69 and under 60 years, respectively.

\section{COVID-19 cases, hospitalisations, ICU admissions and deaths by geographical macro area}

We observed large differences between observed and expected cumulative rates for the four studied outcomes by geographical macro area according to their vaccination coverage (Figures 2 and 3). Areas that achieved high vaccination coverage (around 90\%) by the end of June in those aged 80 years and older (NorthEast, North-West and Centre) already had an estimated percentage of averted events for all outcomes together of over $50 \%$ in the period between April and June.

Without vaccination, between July and September, the overall expected mortality rate for those aged 80 years and older would have been 224 (range: 128-669) per 100,000 vs the observed rate of 32 per 100,000 during the same months (Figure 3). In the South and Islands we observed the lowest difference between the expected, 157 (range: 117-233) and the observed mortality rate, 52; whereas in the Centre we observed the largest difference, 332 (range: $170-1,170$ ) vs 27 . In the same period, for people aged 60-69 and 70-79 years in all the geographical areas, we estimated a percentage of averted hospitalisations and ICU admissions 


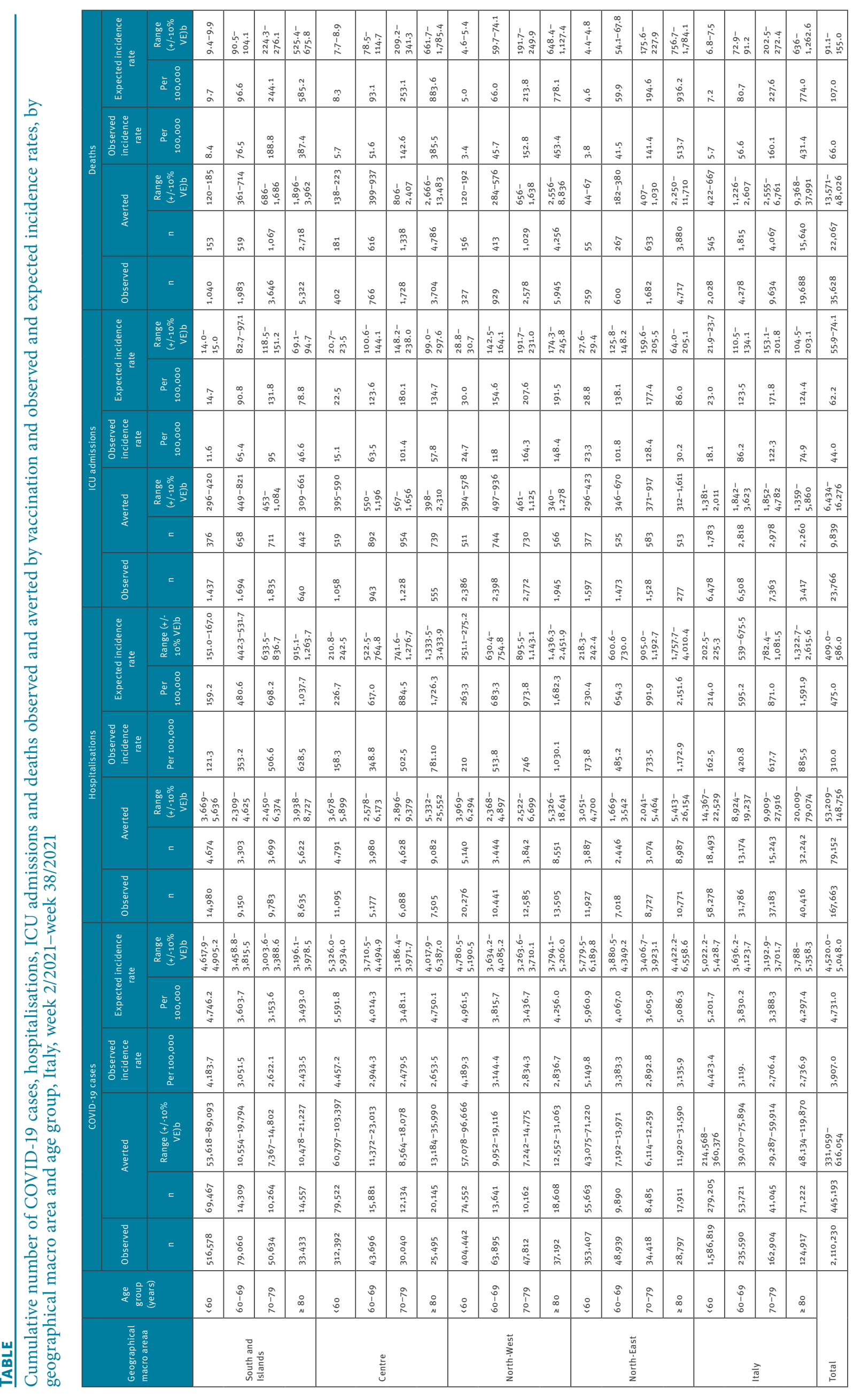


Cumulative and expected incidence rate (A) and cumulative and expected hospitalisation rate (B) with uncertainty ranges ${ }^{\mathrm{a}}$, by period, age group and geographical macro area, Italy, week 2/2021-week 38/2021

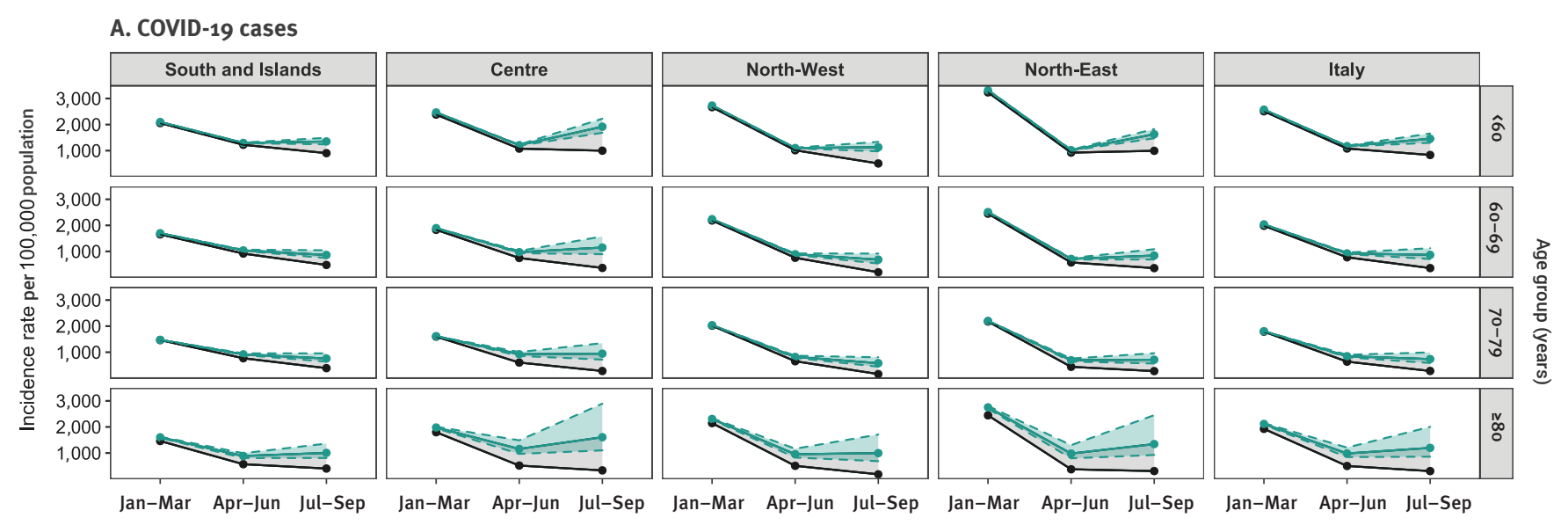

B. Hospitalisations
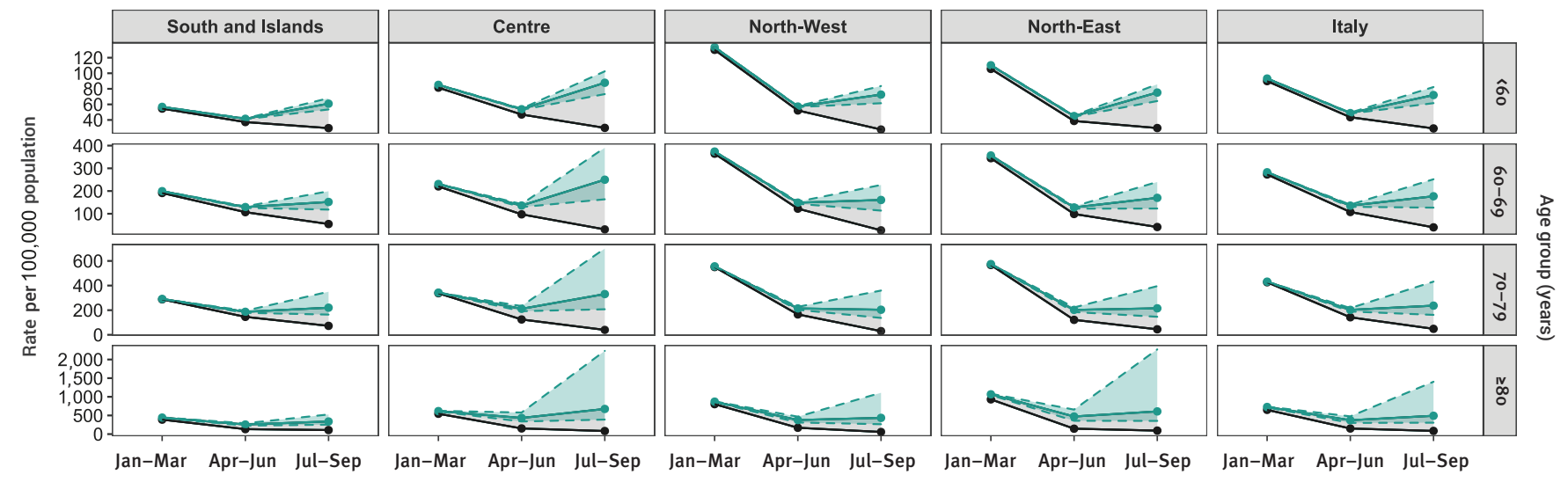

2021

Observed

COVID-19: coronavirus disease; ICU: intensive care unit.

${ }^{a}$ Represent results of the sensitivity analysis, with $+/-10 \%$ vaccine effectiveness.

ICU: intensive care unit.

higher than $60 \%$. Furthermore, for those aged under 60 years, the observed mortality rate and the observed hospital rate was less than half of the expected one by week 38 at the end of September, in all the geographical areas.

Overall, we estimated that $74 \%$ (range: $72-77$ ), $70 \%$ (range: $66-80$ ), 75\% (range: $71-82$ ) and 62\% (range: 55-78) of cases, hospitalisations, ICU admissions and deaths were, respectively, averted between July and September, given that the average full vaccination coverage at the end of September was higher than $60 \%$ in all age groups. Indeed in this period $48 \%$ (range: 40-57), 73\% (range: 63-85), 78\% (range: 68-86) and $83 \%$ (range: $73-93$ ) of the expected cases, hospitalisations, ICU admissions and deaths were averted, respectively.

\section{Ethical statement}

The dissemination of COVID-19 surveillance data was authorised by the Italian Presidency of the Council of Ministers on 27 February 2020 (Ordinance number 640).

\section{Discussion}

The pace of the roll-out of COVID-19 mass vaccination varied by age group and across geographical macro areas in Italy, particularly in people aged 80 years and older, and influenced the magnitude of prevented infections, hospitalisations, ICU admissions and deaths. The South-Islands experienced less averted events than other macro areas mainly because of a slower vaccination uptake in those at higher risk and the high incidence of COVID-19 cases observed during the tourist season (July-August). Rates of expected and observed events for all four outcomes started to diverge in the 


\section{FIGURE 3}

Cumulative and expected ICU admission rate (A) and cumulative and expected mortality rate (B) with uncertainty ranges ${ }^{\mathrm{a}}$, by period, age group and geographical macro area, Italy, week 2/2021-week 38/2021

A. ICU admissions
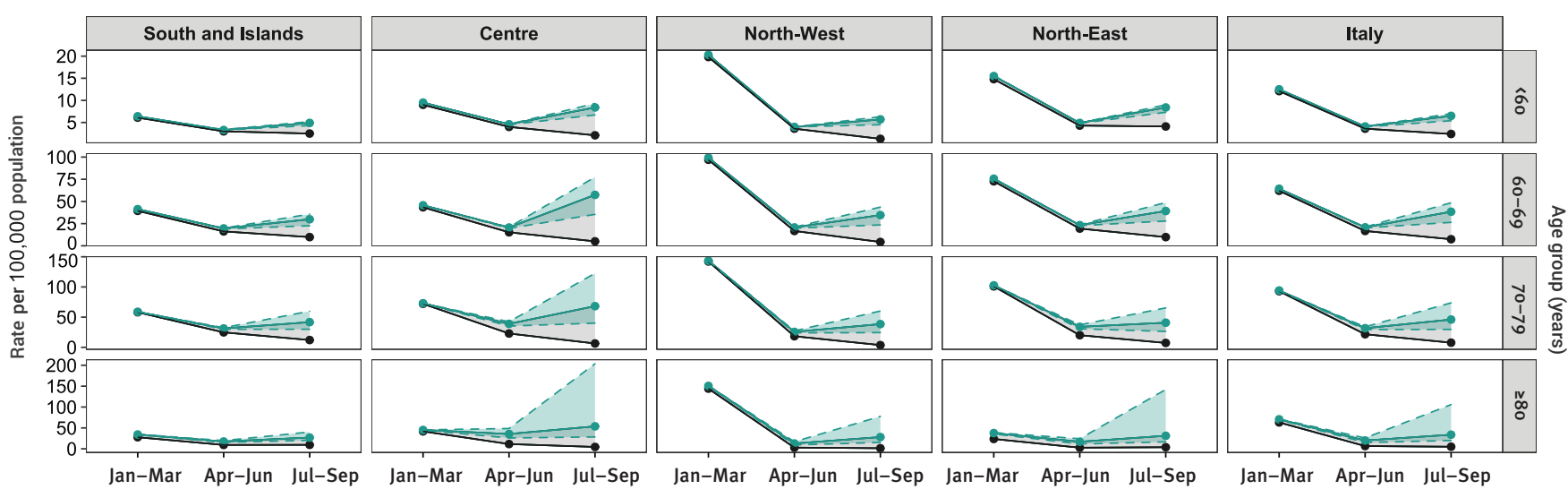

\section{B. Mortality}
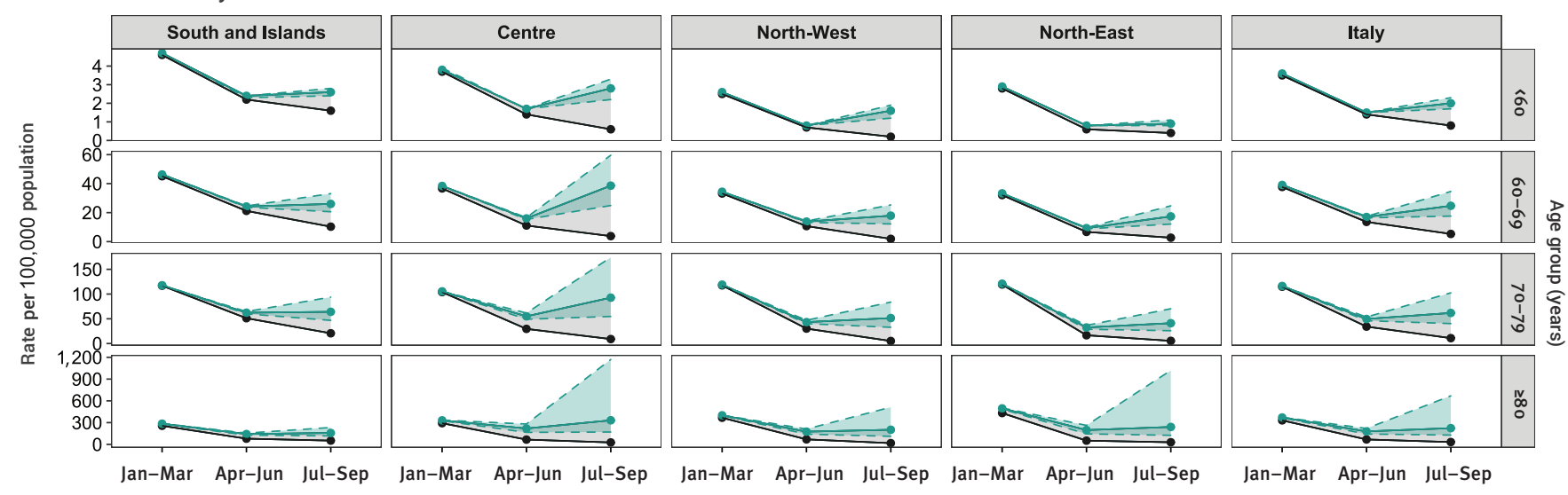

2021

Expected nobserved

COVID-19: coronavirus disease; ICU: intensive care unit.

${ }^{a}$ Represent results of the sensitivity analysis, with $+/-10 \%$ vaccine effectiveness.

ICU: intensive care unit.

period between January and March in those aged 80 years older; and between April and June in the other age groups. Our model estimations show that without vaccination, peaks in hospitalisations, ICU admissions and deaths higher than those observed would have been detected for people aged 80 years and older starting from April and for other age groups from July to September. Overall, the largest proportions of hospitalisations and deaths prevented by the vaccination was observed in the oldest age group (41\%; range $38-53$ and $71 \%$; range $69-79$, respectively), whereas the largest number of averted ICU admissions has been observed in those aged between 60 and 79 years (59\%; range: $52-57$ ).

Our results are consistent with the current literature that demonstrates a positive impact of COVID-19 vaccination in preventing infections [4] and severe disease $[13,15]$, with a larger reduction in the COVID-19 burden in older adults $[16,17]$. Furthermore, previous studies have estimated the number of deaths averted as a result of the vaccination roll-out $[13,15,17,18]$. However, to the best of our knowledge, this is the first study that, exploiting a standard approach, estimates the impact of the COVID-19 vaccinations in terms of prevented events in Italy for all the age groups eligible for vaccination and which analyses geographical differences.

The analysis has several limitations. The method used assumes that vaccination impact is only driven by its direct effects and does not take into account its potential indirect effects such as impact on the overall transmissibility and/or relaxation of restriction measures. 
The proposed calculation may therefore have underestimated the number of avoided events. Moreover, since our approach is not based on a dynamic-transmission model, it is not able to predict future behavioural changes of the population in the counterfactual situation of no-vaccination having been available in 2021, as Italy may have implemented multiple restriction measures over 2021 had the vaccines not been available. Although we performed a sensitivity analysis to determine how different values of VE affect the estimates, we did not take into account other factors that have been found to influence VE, such as the vaccine type [19]. Finally, concurrent with the start of the vaccination roll-out, various non-pharmaceutical interventions were introduced to control the spread of the virus. Both the measures and the vaccination uptake are likely to have had an impact on the incidence of COVID-19 cases, hospitalisations, ICU admissions and deaths.

\section{Conclusion}

Our findings show a positive impact of the COVID-19 vaccination programme in Italy, and suggest that the rapid vaccination of high-risk groups has prevented a considerable number of severe COVID-19 outcomes. Averted hospitalisations and ICU admissions ranged between 53,209 and 148,756 and 6,434 and 16,276, respectively, and for deaths averted the range was 13,571-48,026. Geographical areas that achieved high vaccination rates faster were able to prevent a larger number of hospitalisations, ICU admissions and deaths over the summer months.

\section{Acknowledgements}

Members of the Italian Integrated Surveillance of COVID-19 study group

ISS coordination group: Antonino Bella, Stefano Boros, Marco Bressi, Alessandra Ciervo, Fortunato (Paolo) D’Ancona, Martina Del Manso, Corrado Di Benedetto, Massimo Fabiani, Antonietta Filia, Daniele Petrone, Patrizio Pezzotti, Maria Cristina Rota, Chiara Sacco, Paola Stefanelli, Matteo Spuri, Marco Tallon, Alberto Mateo Urdiales, Maria Fenicia Vescio

Regional representatives: Antonia Petrucci (Abruzzo); Michele La Bianca (Basilicata); Anna Domenica Mignuoli (Calabria); Pietro Buono (Campania); Erika Massimiliani (Emilia-Romagna); Fabio Barbone (Friuli Venezia Giulia); Francesco Vairo (Lazio); Camilla Sticchi (Liguria); Danilo Cereda (Lombardia); Lucia Di Furia (Marche); Raffaele Malatesta (Molise); Pierpaolo Bertoli (P.A. Bolzano); Pier Paolo Benetollo (P.A. Trento); Chiara Pasqualini (Piemonte); Lucia Bisceglia (Puglia); Maria Antonietta Palmas (Sardegna); Salvatore Scondotto (Sicilia); Emanuela Balocchini (Toscana); Anna Tosti (Umbria); Mauro Ruffier (Valle D’Aosta); Filippo Da Re (Veneto).

Funding statement: No funding outside the routine institutional funds were used to carry out this work.

\section{Conflict of interest}

None declared.
Authors' contributions

PP, FR; AMU and CS designed the paper. AB, MDM, MFV and $M B$ retrieved and linked databases. CS, supported by DP and MS, carried out the analysis. CS, AMU, MF, and PP wrote the manuscript, which was then reviewed and approved by the other authors.

\section{References}

1. Agenzia Italiana del Farmaco (AIFA). Vaccini COVID-19. [COVID-19 Vaccines]. Rome: AIFA. [Accessed: 1 Oct 2021]. Italian. Available from: https://www.aifa.gov.it/ vaccini-covid-19

2. Mateo-Urdiales A, Spila Alegiani S, Fabiani M, Pezzotti P, Filia A, Massari M, et al. Risk of SARS-CoV-2 infection and subsequent hospital admission and death at different time intervals since first dose of COVID-19 vaccine administration, Italy, 27 December 2020 to mid-April 2021. Euro Surveill. 2021;26(25):2100507. https://doi.org/10.2807/1560-7917. ES.2021.26.25.2100507 PMID: 34169819

3. Istituto Superiore di Sanità (ISS). Epicentro. Impact of COVID-19 vaccination on the risk of SARS-CoV-2 infection and hospitalization and death in Italy (27.12.2020 - 29.08.2021). Rome: ISS; 30 Sep 2021. Italian. Available from: https:// www.epicentro.iss.it/vaccini/pdf/report-valutazione-impattovaccinazione-covid-19-6-ott-2021.pdf

4. Harder T, Koch J, Vygen-Bonnet S, Külper-Schiek W, Pilic A, Reda S, et al. Efficacy and effectiveness of COVID-19 vaccines against SARS-CoV-2 infection: interim results of a living systematic review, 1 January to 14 May 2021. Euro Surveill. 2021;26(28):2100563. https://doi.org/10.2807/1560-7917. ES.2021.26.28.2100563 PMID: 34269175

5. Ministero della Salute. Piano strategico per la vaccinazione anti-SARS-CoV-2/COVID-19. [Strategic plan for SARS-CoV-2 COVID-19 vaccination]. Rome: Ministero della Salute; 2020. Italian. Available from: https://www.trovanorme.salute.gov.it/ norme/renderNormsanPdf?anno $=2021 \& \operatorname{codLeg}=78657 \&$ parte $=1 \&$ serie $=$ null

6. Eurostat. NUTS, Nomenclature of territorial units for statistics. Luxembourg: Eurostat. [Accessed: 25 Oct 2021]. Available from: https://ec.europa.eu/eurostat/web/nuts/background

7. Lopez Bernal J, Andrews N, Gower C, Robertson C, Stowe J, Tessier E, et al. Effectiveness of the Pfizer-BioNTech and Oxford-AstraZeneca vaccines on covid-19 related symptoms, hospital admissions, and mortality in older adults in England: test negative case-control study. BMJ. 2021;373(1088):n1088. https://doi.org/10.1136/bmj.n1088 PMID: 33985964

8. Baden LR, El Sahly HM, Essink B, Kotloff K, Frey S, Novak R, et al. Efficacy and Safety of the mRNA-1273 SARS-CoV-2 Vaccine. N Engl J Med. 2021;384(5):403-16. https://doi.org/10.1056/ NEJMoa2035389 PMID: 33378609

9. Riccardo F, Ajelli M, Andrianou XD, Bella A, Del Manso M, Fabiani M, et al. Epidemiological characteristics of COVID-19 cases and estimates of the reproductive numbers 1 month into the epidemic, Italy, 28 January to 31 March 2020. Euro Surveill. 2020;25(49):2000790. https://doi.org/10.2807/1560-7917. ES.2020.25.49.2000790 PMID: 33303064

10. Governo Italiano. Presidenza del Consiglio dei Ministri. Data Repository. [Accessed: 11 Nov 2021]. Italian. Available from: https://github.com/italia/covid19-opendata-vaccini

11. Bonmarin I, Belchior E, Lévy-Bruhl D. Impact of influenza vaccination on mortality in the French elderly population during the 2000-2009 period. Vaccine. 2015;33(9):1099101. https://doi.org/10.1016/j.vaccine.2015.01.023 PMID: 25604800

12. Machado A, Mazagatos C, Dijkstra F, Kislaya I, Gherasim A, McDonald SA, et al. Impact of influenza vaccination programmes among the elderly population on primary care, Portugal, Spain and the Netherlands: $2015 / 16$ to 2017/18 influenza seasons. Euro Surveill. 2019;24(45):1900268. https://doi.org/10.2807/1560-7917.ES.2019.24.45.1900268 PMID: 31718740

13. Public Health England (PHE). Impact of COVID-19 vaccines on mortality in England - December 2020 to March 2021. London: PHE; 2021. Available from: https://assets.publishing. service.gov.uk/government/uploads/system/uploads/ attachment_data/file/972592/COVID-19_vaccine_impact_on mortality_240321.pdf

14. R Core Team. R: A language and environment for statistical computing. Vienna: R Foundation for Statistical Computing. [Accessed: 25 Oct 2021]. Available from: https://www.Rproject.org/. 
15. Galvani A, Moghadas SM, Schneider EC. Deaths and hospitalizations averted by rapid US vaccination rollout. The Commonwealth Fund. Issue Briefs.2021; (July):7.

16. Hyams C, Marlow R, Maseko Z, King J, Ward L, Fox K, et al. Effectiveness of BNT162b2 and ChAdOx1 nCoV-19 COVID-19 vaccination at preventing hospitalisations in people aged at least 80 years: a test-negative, case-control study. Lancet Infect Dis. 2021;21(11):1539-48. https://doi.org/10.1016/S14733099(21)00330-3 PMID: 34174190

17. Goldstein JR, Cassidy T, Wachter KW. Vaccinating the oldest against COVID-19 saves both the most lives and most years of life. Proc Natl Acad Sci USA. 2021;118(11):e2026322118. https://doi.org/10.1073/pnas.2026322118 PMID: 33632802

18. Victora PC, Castro PMC, Gurzenda S, Medeiros AC, França GVA Barros PAJD. Estimating the early impact of vaccination against COVID-19 on deaths among elderly people in Brazil: Analyses of routinely-collected data on vaccine coverage and mortality. EClinicalMedicine. 2021;38:101036. https://doi.org/10.1016/j. eclinm.2021.101036 PMID: 34308302

19. Lopez Bernal J, Andrews N, Gower C, Gallagher E, Simmons R, Thelwall S, et al. Effectiveness of Covid-19 vaccines against the B. 1.617. 2 (Delta) variant. N Engl J Med. 2021;385(7):585-94. https://doi.org/10.1056/NEJMoa2108891 PMID: 34289274

\section{License, supplementary material and copyright}

This is an open-access article distributed under the terms of the Creative Commons Attribution (CC BY 4.0) Licence. You may share and adapt the material, but must give appropriate credit to the source, provide a link to the licence and indicate if changes were made.

Any supplementary material referenced in the article can be found in the online version.

This article is copyright of the authors or their affiliated institutions, 2021. 